\title{
PARASITES ET PHORÉTIQUES DE COLÉOPTÈRES ET DE MYRIAPODES DE RICHELIEU (Indre-et-Loire)
}

\author{
Par Jean THEODORIDĖS
}

Lors de cinq séjours à la Station expérimentale de Richelieu (Indreet-Loire) (juillet 1947, avril 1949, août 1949, septembre 1951, avril 1955). nous avons récolté un certain nombre d'organismes parasites et phorétiques de Coléoptères et accessoirement de Myriapodes.

La plupart de ceux-ci ont été mentionnés de Richelieu dans un travail antérieur (Théodoridès 1955) et seront simplement cités ci-dessous, leur nom étant précédé d'un astérisque et suivi de l'indication de la page où l'espèce est décrite ou signalée.

Nous étudierons par contre plus en détail d'autres espèces trouvées lors de notre séjour de 1955, ou récoltées en 1956 par notre ami Yves Golvan.

\section{A. Parasites et Phorétiques de Coléoptères}

\section{Eugrégarines}

Ces Protistes furent tous trouvés dans l'intestin moyen des insectes étudiés.

\section{Gregarinidæ}

* 1. Gregarina acuta Léger.

Hôte : Trox hispidus (Pontopp.), 11 août 1951 (1), (op. cit., p. 60).

* 2. Gregarina munieri (A. Schneider).

Hôtes : Timarcha goettingensis L., Chrysolina menthastri Suff., Chrysolina staphylea L., 3 août 1951 (op. cit., p. 64-65).

(1) Lorsqu'aucune indication de localité n'est indiquée, cela signifie que les hôtes provenaient du Domaine universitaire de Richelieu. Nous remercions ici MM. A. Balachowsky, H. Bertrand et F. I. Van Emden pour la détermination de certains Coléoptères. 


\section{Gregarina typographi Fuchs.}

Hôte : Ips sexdentatus Boerner (A. Balachowsky, det.), 11 septembre 1956 (Y. Golvan, leg.).

Cette espèce, décrite par Fuchs (1915) chez Ips typographus L., a été retrouvée chez le même hôte en Tchécoslovaquie par Weiser (1954, 1955).

Les exemplaires de Richelieu correspondent exactement à la description et aux figures de ces auteurs et nous permettent de préciser les dimensions des divers stades de cette Grégarine qui ne sont pas données par Fuchs et Weiser.

Céphalins: Les plus jeunes stades observés mesurent environ $25 \mu$ et ont un volumineux noyau cylindrique d'environ $10 \mu$ de diamètre; l'épimérite n'est pas visible, étant enfoncé dans les cellules épithéliales de l'intestin de l'hôte.

Chez des céphalins détachés de ce dernier, on voit l'épimérite qui a la forme d'un prolongement arrondi, dont la base plus large fait suite au protomérite (fig. 1, A).

Associations: Ces stades ne sont pas figurés par Fuchs. Deux associations observées mesurent
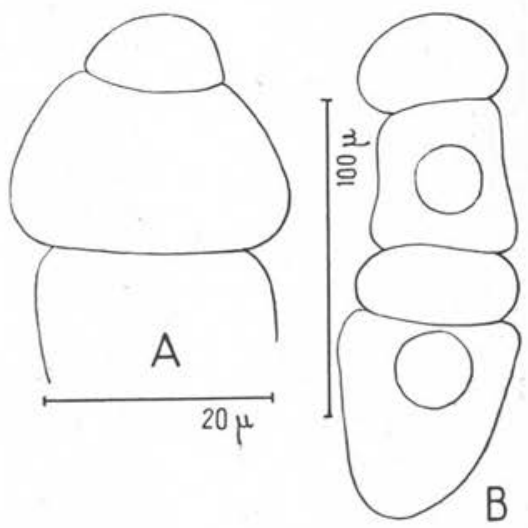

FIG. 1. - Gregarina typographi Fuchs. A, Epimérite d'un céphalin âgé. B, Association. respectivement : primites : 75 et $130 \mu$, satellites : 85 et $130 \mu$; le noyau cylindrique a environ $20 \mu$ de diamitre.

Sporadins libres : Nous avons observé des jeunes sporadins mesurant respectivement $45,50,60,80,90 \mu$. Chez les formes les plus âgées, le protomérite est hémisphérique et plus large que le deutomérite.

Gregarina typographi est une espèce nouvelle pour la France, et Ips sexdentatus, un hôte inédit.

\section{Gregarina sp.}

Hôte: larve de Prionychus ater F. (F. I. Van Emden, det.), 12 avril 1955.

Nous préférons ne pas nommer cette espèce probablement inédite trouvée chez cet Alléculide, car nous n'avons observé que des 
associations. Trois de celles-ci ont les dimensions approximatives suivantes :

Association 1 Association 2 Association 3

$\begin{array}{lrrrr} & \overline{-} & -\overline{-} & - \\ \text { Longueur totale . . } & 140 \mu & 180 \mu & 225 \mu \\ \text { Longueur primite. } & 70 \mu & 90 \mu & 120 \mu \\ \text { Longueur satellite . } & 70 \mu & 90 \mu & 105 \mu \\ \text { Diamètre noyaux . . } & 10 \mu & 20 \mu & 20-25 \mu\end{array}$

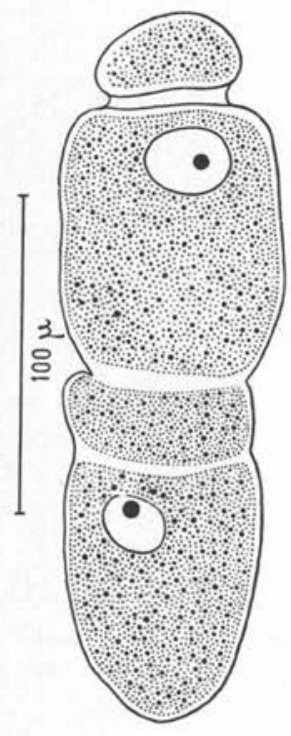

FIg. 2. - Gregarina sp Association

Le protomérite du primite est en forme de bouton légèrement aplati; celui du satellite peut être presque quadrangulaire ; dans l'association mesurant $225 \mu$, on voit très bien les nucléoles (fig. 2).

L'entocyte de cette espèce comprend deux sortes de granulations bien visibles à un fort grossissement.

\section{Actinocephalidae}

Actinocephalinae.

* 5. Actinocephalus conicus (Dufour).

Hôtes : Dorcus parallelipipedus L., 3 et 13 août 1951 (op. cit., p. 76) ; larve de Ludius ferrugineus L. (F. I. Van Emden, det.), 12 avril 1955.

La mise en évidence de cette Grégarine chez ce dernier hôte qui est un Elatéride est assez inattendue, car jusqu'ici elle semblait strictement inféodée aux Lucanides.

Il s'agit là d'une infestation écologique accidentelle, les larves de Ludius provenant d'une souche de saule qui hébergeait des larves de Dorcus (négatives en Grégarines).

De tels cas ont déjà été signalés en ce qui concerne les Eugrégarines (cf. Théodoridès, 1955, p. 250).

* 6. Actinocephalus conicus (Dufour), var. magna Théodoridès.

Hôte : Lucanus cervus L., 13 août 1951 (op. cit., p. 76).

7. Legeria agilis (Schneider).

Hôte: larve de Dytiscus semisulcatus Mull. (H. Bertrand, det.), 13 avril 1955. 


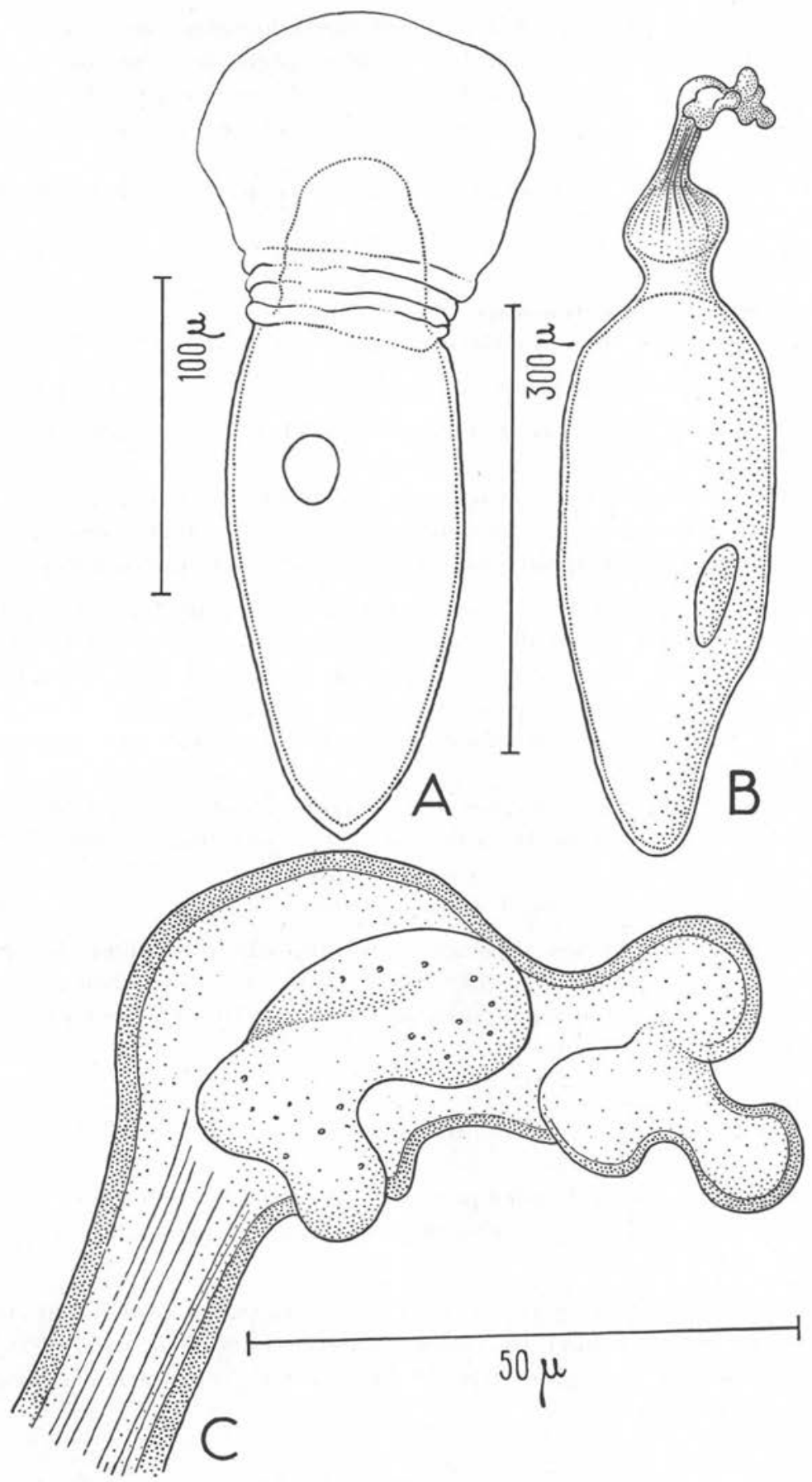

FIG. 3. - A, Legeria agilis (Schneider), sporadin typique ;

B, Actinocéphalide (?) indéterminé, céphalin ; C, Id. détail de l'épimérite. 
Nous avons observé chez cet hôte des sporadins de cette Grégarine conformes en tous points à la description de Schneider (1876) et caractérisés par leur protomérite dilaté en massue, le septum étant « courbé en manière de voûte »(Schneider) dans ce dernier (fig. 3, A).

La longueur de cinq exemplaires mesurés était d'environ 130, 140, 270,350 et $400 \mu$.

Acanthosporinae.

* 8. Ancyrophora cervicornis Théodoridès.

Hôte : Silpha carinata Herbst, 7 août 1951 (op. cit., p. 72).

\section{Actinocéphalide (?) indéterminé.}

Hôte : larve de Dytiscus semisulcatus (H. Bertrand, det.), 13 avril 1955.

Chez l'hôte qui hébergeait des sporadins de Legeria agilis, se trouvaient des stades d'une autre Eugrégarine fort curieuse, qui doit probablement appartenir au groupe des Actinocéphalides.

Céphalin (fig. 3, B) : Il mesure environ $500 \mu$ de long et l'épimérite est constitué par deux volumineuses papilles en forme de «bonnet phrygien 》 (dont l'une était quelque peu étirée dans la préparation étudiée) (fig. 3, C), portées par une hampe à épicyte bien marqué et à protoplasme finement strié, d'environ $45 \mu$ de long et de moins de $15 \mu$ de large.

Les stries protoplasmiques sont encore visibles dans le protomérite sphérique à sa partie antérieure et séparé du deutomérite par un rétrécissement bien marqué (fig. $3, \mathrm{~B}$ ).

Le noyau est ovalaire et mesure environ $70 \mu$.

Kystes : Les kystes prélevés dans l'intestin postérieur de l'hôte mesurent les uns environ $225 \mu$ de diamètre, les autres $300 \mu$ et possèdent une gangue d'une épaisseur d'environ $40 \mu$ chez les premiers et $60 \mu$ chez les seconds.

\section{Discussion}

Nous avions tout d'abord pensé que ces stades (céphalin et kystes) étaient ceux de Legeria agilis, mais il s'agit très probablement d'une toute autre espèce, car :

1) La conformation du septum du céphalin est tout à fait différente de chez L. agilis où, comme nous venons de le voir, cette région séparant le deutomérite du protomérite est courbée en voûte dans ce dernier. 
2) Pour Blunck (1923), chez L. agilis, l'épimérite et le protomérite ne feraient qu'un organe ( Epi-Protomerit $»)$, et c'est cette partie de la Grégarine qui s'insérerait entre les cellules épithéliales de l'intestin de l'hôte.

Cette interprétation de l'auteur allemand demanderait à être vérifiée, mais on peut en tout cas dire que l'on n'a jamais décrit chez L. agilis un épimérite constitué par deux papilles portées par un col.

3) Toujours selon Blunck, le diamètre des kystes de L. agilis serait de $800 \mu(2)$, mais cet auteur a également observé, chez des larves de Dytiscus marginalis, des kystes plus petits, de $225 \mu$ de diamètre, avec une gangue de $40 \mu$ d'épaisseur, correspondant bien à ceux trouvés à Richelieu chez la larve de Dytiscus semisulcatus.

Tout ceci autorise à penser que les larves de Dytiscus semisulcatus peuvent être parasitées par deux Eugrégarines : Legeria agilis et une autre espèce encore mal connue, qui doit également être un Actinocéphalide. A notre connaissance, on n'avait pas encore signalé de Grégarines chez ce Dytiscide.

\section{Stylocephalidae}

\section{Stylocephalus oblongatus (Hammerschmidt).}

Hôte : Opatrum sabulosum L., 4 avril 1956 (Y. Golvan, leg.).

Ce Ténébrionide est un des hôtes réguliers de cette espèce.

\section{Nématodes}

\section{Rhabditidae}

1) EsPÈCES PHORÉtiQues :

Les larves de ces Vers se rencontrent dans le segment génital des Coléoptères où elles trouvent un refuge contre la dessiccation.

\section{* 1. Rhabditis stammeri Völk.}

Hôtes : Necrophorus vestigator Hersch., N. vespillo L., N. humator F., mai 1949 (op. cit., p. 113).

\section{* 2. Rhabditis sp.}

Hôte : Necrophorus vespilloides Herbst, 6 à 15 août 1951 (op. cit., p. 114).

\section{* 3. Diplogaster hirschmannce Sachs.}

Hôtes : Geotrupes stercorarius L., G. mutator Marsh, G. stercorosus Scriba, G. vernalis L., avril 1949.

(2) Schneider (1876) ne donne pas la dimension des kystes de L. agilis, que l'on ne trouve pas indiquée non plus par les autres auteurs qui ont revu cette Grégarine. 
Cette espèce se rencontre presque constamment dans le segment génital des Géotrupes.

2) EsPèces parasites :

Les larves se rencontrent dans la cavité générale de l'hôte.

* 4. Rhabditis insectivora Körner.

Hôte : Dorcus parallelipipedus L., 3 et 13 août 1951 (op. cit., p. 118).

\section{Oxyuroidea, Thelastomatidae}

Ce sont des parasites inoffensifs de l'intestin postérieur.

5. Thelastoma macramphidum Christie, var. gallica Théodoridès.

Hôte : larve de Cetonia aurata L. (F. I. Van Emden, det.), 14 avril 1955 .

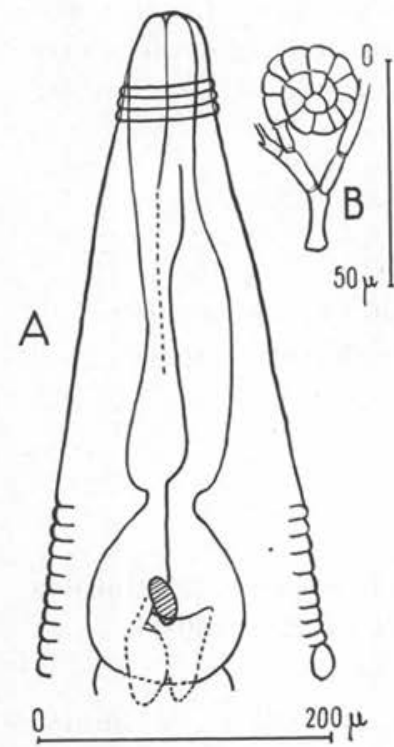

Fig. 4. - Thelastoma macramphidum Christie var. gallica Théodoridès. A, Aspect de l'osophage d'une femelle en vue latérale ; $\mathrm{B}$, Champignon observé dans le contenu intestinal.

Nous avons décrit cette variété chez des larves de Scarabéides [Phyllognathus (3), Cetonia, Potosia], des Pyrénées-Orientales (Théodoridès, 1955, page 129).

Les exemplaires récoltés à Richelieu correspondent à notre diagnose et se rapprochent parfois de la forme typique décrite par Christie (1931), le pore excréteur étant alors au niveau de la partie postérieure du bulbe.

Ces Nématodes étaient tous des femelles à maturité, dont la longueur totale variait entre $1,7 \mathrm{~mm}$. et $2,6 \mathrm{~mm}$. L'œsophage mesurait entre 225 et $450 \mu$ suivant l'âge des individus et le corpus œsophagien présentait parfois en vue latérale un aspect dissymétrique, étant renflé d'un côté (fig. 4, A). On remarque dans l'intestin la présence de fragments de Champignons dont se nourrit le Nématode (fig. $4, \mathrm{~B}$ ).

La distance entre l'anus et l'extrémité postérieure varie de 330 à $400 \mu$ et celle

(3) L'indication d'hôte : Orgctes nasicornis (L.) donnée dans notre ouvrage (Théodoridès, 1955, p. 129) est erronée et doit être remplacée par Phyllognathus silenus (F.), le $\mathrm{D}^{\mathrm{r}}$ Van Emden ayant entre temps réexaminé nos larves, qu'il avait tout d'abord rapportées au premier de ces deux Scarabéides Dynastides. 
entre la vulve et l'extrémité antérieure de $930 \mu$ à 1,5 mm. Les œufs utérins mesurent environ $70 \times 45 \mu$.

Cetonia aurata est un hôte nouveau pour ce Nématode.

\section{Acariens}

De nombreux Acariens phorétiques ou parasites de Coléoptères ont été récoltés à Richelieu.

\section{Parasitiformes}

A) Gamasides :

Toutes ces espèces sont phorétiques.

\section{Macrochelidae}

* 1. Macrocheles sp.

Hôtes : Necrophorus humator, N. vespillo, 4 mai 1949 et 27 avril 1949 (op. cit., p. 158).

* 2. Coprholaspis glaber (Müller).

Hôte : Geotrupes vernalis L., 13 août 1951 (op. cit., p. 158).

\section{Poecilochiridae}

* 3. Poecilochirus necrophori Vitzthum.

HôtEs : Necrophorus humator, 7 mai 1949 ; N. vestigator, 25 avril 1949 (op. cit., p. 159).

* 4. Poecilochirus subterraneus Oudemans.

Hôte : Necrophorus humator, 7 mai 1949 (op. cit., p. 159).

\section{Laelaptidae}

* 5. Lasioseius sp.

Hôte : Necrophorus vespillo, 26 avril 1949 (op. cit., p. 161).

B) Uropodina :

\section{Uropodidae}

* 6. Pseudouropoda vegetans Degeneer.

Hôte : Dorcus parallelipipedus, 3 août 1951 (op. cit., p. 162).

C) Trachytina : 


\section{Trachytidae}

\section{* 7. Neoseius novus (Oudemans).}

Hôtes : Necrophorus vespillo, 24-29 avril 1949 ; N. vespilloides, 26 avril 1949 ; Lucanus cervus, 3 août 1951 ; Dorcus parallelipipedus, 13 août 1951 (op. cit., p. 163).

\section{Polyaspidae}

\section{* 8. Polyaspis patavinus Berlese.}

Hôtes : Dorcus parallelipipedus, 3 août 1951 (deutonymphes et femelles) ; Lucanus cervus, 13 août 1951 (deutonymphes) (op. cit., p. 163).

\section{SARCoptiformes}

On trouve dans cet ordre des espèces phorétiques sessiles (hypopes de Tyroglyphides) ou des ectoparasites permanents (Canestriniens).

\section{Tyroglyphidae}

\section{* 9. Histiostoma sp.}

Hôtes : Oeceoptoma thoracicum Ganglb., 7 août 1951 ; Paederus ruficollis $\mathrm{F}$., 25 avril 1949 (op. cit., p. 165).

\section{Caloglyphidae}

* 10. Caloglyphus geotruporum Zachvatkin.

Hôte: Geotrupes stercorosus Scriba, 13 août 1951 (op. cit., p. 165).

\section{Canestriniidae}

Les espèces de cette famille sont toutes exclusivement inféodées à des Coléoptères.

* 11. Canestrinia dorcicola Berlese.

Hôte: Dorcus parallelipipedus, 3 août 1951 (op. cit., p. 167), 12 avril 1955 .

\section{* 12. Paramansia menthastri Cooreman.}

Hôte : Chrysolina menthastri Suff., Braye-sous-Faye, près Richelieu, 16 août 1949 (op. cit., p. 169).

Ce genre, représenté jusqu'ici par cette seule et unique espèce, a été décrit d'après notre matériel par Cooreman (1950); cette espèce semble très rare et n'a été trouvée (en 3 exemplaires) qu'une seule fois chez un seul des 104 Coléoptères de cette espèce examinés. 
* 13. Percanestrinia saetolata Cooreman.

Hôte : Procrustes purpurascens F., 9 août 1951 (op. cit., p. 169).

* 14. Pseudamansia chrysomelinus (C.-L. Koch).

Hôte : Timarcha goettingensis L., 3 août 1951 (op. cit., p. 171).

\section{Linobiidae}

* 15. Linobia coccinella Scopoli.

Hôte : Chrysomela populi L., 11 août 1951 (op. cit., p. 171).

\section{B. Parasites et Phorétiques de Myriapodes}

D'après des recherches préliminaires faites lors de notre cinquième séjour à Richelieu (avril 1955), les Myriapodes semblent être beaucoup moins parasités que les Coléoptères. Nous avons pu cependant observer chez eux les organismes suivants :

\section{Eugrégarines}

Même localisation que chez les Coléoptères (intestin moyen).

\section{Stenophoridae}

\section{Stenophora iulipusilli (Labbé) Crawley (4).}

Hôte : Cylindroiulus londinensis Leach, 12 et 14 avril 1955.

Les sporadins observés mesuraient de 60 à plus de $200 \mu$ de long et présentaient la morphologie caractéristique de cette espèce : protomérite conique et deutomérite de forme cylindrique, arrondi à sa partie postérieure.

Cette espèce n'était jusqu'ici signalée que chez des Iulides des Etats-Unis (cf. Watson, 1916), et sa mise en évidence à Richelieu montre qu'elle existe également en Europe.

\section{Dactylophoridae}

\section{Trichorhynchus pulcher A. Schneider.}

Hôte : Scutigera coleoptrata L., 11 avril 1955.

Cette espèce fut décrite chez cet hôte par Schneider (1882).

Nous avons observé des céphalins mesurant de 450 à $600 \mu$ avec l'épimérite caractéristique en forme de languette qui peut atteindre $120 \mu$ de longueur.

(4) Nous remercions ici Mlle O. Tuzet et M. R. Ormières, qui ont bien voulu examiner nos préparations et nous confirmer la détermination de cette espèce et de C. lutea. Les Myriapodes ont été déterminés par M. M. Darbois, que nous remercions également. 
Dans un céphalin de $450 \mu$, le noyau a environ $50 \mu$ de diamètre et présente un gros nucléole.

Les kystes sphériques ont un diamètre d'environ $350 \mu$, pouvant atteindre parfois $385 \mu$, comme viennent de le signaler Tuzet et Ormières (1956).

\section{Cnemidosporidae}

3. Cnemidospora lutea A. Schneider.

HôtE : Glomeris marginata Villers, 14 avril 1955.

Cette espèce a été décrite par Schneider (1882) chez des Glomeris sp., de Poitiers, localité assez proche de Richelieu.

Les sporadins observés mesuraient de 400 à $550 \mu$ et présentaient la morphologie propre à cette espèce : protomérite subglobuleux et deutomérite cylindrique très allongé.

Rappelons que cette Grégarine avait été revue par Tuzet et Guérin (1946), qui l'avaient trouvée précisément chez Glomeris marginata, ce qui leur avait permis de compléter la description des stades jeunes.

\section{Nématodes}

\section{Oxyuroidea, Thelastomatidae}

\section{Thelastoma galliardi Dollfus.}

Hôtes : Cylindroiulus londinensis Leach, 14 avril 1955 ; Glomeris marginata Villers, 15 avril 1955.

Les exemplaires recueillis dans l'intestin postérieur de ces deux Diplopodes correspondent, à quelques différences près dans leurs dimensions, à cette espèce décrite de Richelieu chez un Glomeris par Dollfus (1952).

Voici les dimensions approximatives de deux femelles provenant de chacun des deux hôtes :

Chez C. londinensis Chez G. marginata

\begin{tabular}{|c|c|c|}
\hline Longueur totale $\ldots \ldots \ldots$ & $1 \mathrm{~mm} .6$ & $3 \mathrm{~mm} .12$ \\
\hline Largeur moyenne ....... & $100 \mu$ & $125 \mu$ \\
\hline $\begin{array}{l}\text { Queue (distance anus-extré- } \\
\text { mité postérieure) } \ldots \ldots \ldots\end{array}$ & $240 \mu$ & $350 \mu$ \\
\hline $\begin{array}{l}\text { Longueur du corpus œeso- } \\
\text { phagien } \ldots \ldots \ldots \ldots \ldots \ldots\end{array}$ & $280 \mu$ & $275 \mu$ \\
\hline $\begin{array}{l}\text { Largeur du corpus }{ }^{2} \text { pso- } \\
\text { phagien } \ldots \ldots \ldots \ldots \ldots \ldots\end{array}$ & $30 \mu$ & 25 \\
\hline
\end{tabular}




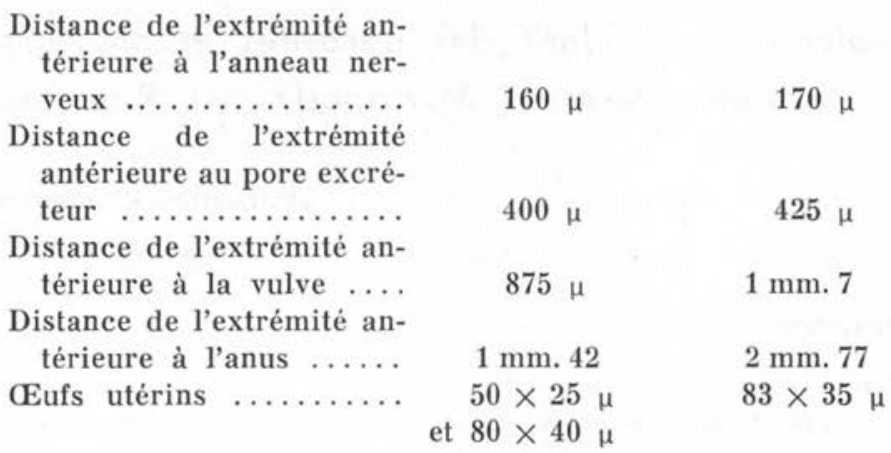

Comme il était prévisible, les dimensions de l'exemplaire provenant de G. marginata se rapprochent de celles données par Dollfus (op. cit.) (5).

\section{Spiruroidea}

Dollfus (op. cit., p. 158, note 1) signale avoir observé chez les Glomeris de Richelieu des larves de Spiruroidea enkystées dans la cavité générale.

Nous en avons également trouvé chez un exemplaire de Glomeris marginata examiné le 15 avril 1955.

\section{Acariens}

\section{Histiostoma sp.}

\section{Caloglyphus sp.}

Hôte : Polydesmus complanatus L., 15 avril 1955.

Des deutonymphes de ces deux genres de Sarcoptiformes (obligeamment déterminées par M. J. Cooreman, de Bruxelles, que nous remercions ici) ont été trouvées dans le tube digestif de ce Diplopode.

Cette curieuse localisation avait été déjà remarquée par Mlle J.-F. Manier (1950 et in litt., 1956) chez des Glomeris et Iules de la garrigue montpelliéraine et s'explique si on considère que ces minuscules formes larvaires pratiquant la phorésie chez le Diplopode peuvent être accidentellement ingérées par celui-ci, surtout lorsqu'elles sont très abondantes dans la région céphalique.

(5) Dans le mémoire de R.-Ph. Dollfus (op. cit., p. 157), on trouve comme dimension de la queue, chez une femelle de Th. galliardi, le chiffre de $292 \mu$; il s'agit là d'une erreur typographique, et $M$. Dollfus a bien voulu revoir son matériel et nous préciser que cette partie du corps du Nématode mesurait en fait environ $370 \%$ 
Tableau récapitulatif des parasites et phorétiques de Coléoptères et Myriapodes de Richelieu

\section{Hôtes}

I. Coléoptères.

1. Carabiques :

Procrustes purpurascens.

2. Dytiscides :

Dytiscus semisulcatus (larve).

3. Silphides :

Necrophorus humator.

Necrophorus vespilloides.

Necrophorus vespillo.

Necrophorus vestigator.

Oeceoptoma thoracicum.

Silpha carinata.

4. Staphylinides :

Paederus ruficollis.

5. Lucanides :

Lucanus cervus.

Dorcus parallelipipedus.

\section{Parasites et phorétiques}

Percanestrinia saetolata.

Legeria agilis.

? Actinocéphalide.

Rhabditis stammeri.

Macrocheles sp.

Poecilochirus necrophori.

Poecilochirus subterraneus.

Rhabditis sp.

Neoseius novus.

Rhabditis stammeri.

Macrocheles sp.

Lasioseius sp.

Neoseius novus.

Rhabditis stammeri.

Poecilochirus necrophori.

Histiostoma sp.

Ancyrophora cervicornis.

Histiostoma sp.

Actinocephalus conicus var. magna.

Neoseius novus.

Polyaspis patavinus.

Actinocephalus conicus.

Rhabditis insectivora.

Coleolaelaps campestris.

Pseudouropoda vegetans.

Neoseius novus.

Polyaspis patavinus.

Canestrinia dorcicola. 
6. Trogides :

Trox hispidus.

Gregarina acuta.

7. Scarabéides :

Geotrupes stercorosus.

Geotrupes stercorarius.

Geotrupes mutator.

Geotrupes vernalis.

Cetonia aurata (larve).

8. Elatérides :

Ludius ferrugineus (larve).

9. Alléculides :

Prionychus ater (larve).

10. Ténébrionides :

Opatrum sabulosum.

11. Chrysomélides :

Timarcha goettingensis.

Chrysolina staphylea.

Chrysolina menthastri.

Chrysomela populi.

12. Scolytides :

Ips sexdentatus.

II. Myriapodes.

\section{ChILOpodes}

1. Scutigérides :

Scutigera coleoptrata.

\section{Diplopodes}

2. Glomérides :

Glomeris marginata.

3. Polydesmides :

Polydesmus complanatus.

4. Iulides :

Cylindroiulus londinensis.

Diplogaster hirschmannæ. Caloglyphus geotruporum. Diplogaster hirschmannæ. Diplogaster hirschmannæ. Diplogaster hirschmannæ. Coprholaspis glaber. Thelastoma macramphidum var. gallica.

Actinocephalus conicus.

Gregarina sp.

Stylocephalus oblongatus.

Gregarina munieri.

Pseudamansia chrysomelinus.

Gregarina munieri.

Gregarina munieri.

Linobia coccinella.

Gregarina typographi.

Trichorhynchus pulcher.

Cnemidospora iutea.

Thelastoma galliardi

larves de Spiruroidea.

Histiostoma sp.

Caloglyphus sp.

Stenophora iulipusilli. Thelastoma galliardi. 
En conclusion, nous avons pu mettre en évidence à Richelieu une quarantaine d'espèces de parasites et phorétiques de Coléoptères et de Myriapodes, dont nous donnons pour terminer la récapitulation sous forme d'un tableau hôtes-parasites (p. 500-501) (6).

\section{BiBLIOGRAPHIE}

Blunck (H.), 1923. - Krankheiten, Feinde und Parasiten des Gelbrands. Zool. Anz., 57, 296-328.

Christie (J. R.), 1931. - Some nemic parasites of coleopterous larvæ. J. Agr. Res., 42, 463-482.

Cooreman (J.), 1950. - Etude de quelques Canestriniidæ (Acari) vivant sur des Chrysomelida et sur des Carabidæ (Insecta Coleoptera). Bull. Inst. Roy. Sci. Nat. Belg., XXVI, 33, 54 p.

Dollfus (R.-Ph.), 1952. - Quelques Oxyuroidea de Myriapodes. Ann. Parasit. Hum. Comp., 27, 143-236.

Fuchs (G.), 1915. - Die Naturgeschichte der Nematoden und einiger anderer Parasiten ; 1. des Ips typographicus (sic) L. ; 2. des Hylobins abietis L. Zool. Jahrb. (Abt. Syst.), 38, 109-222.

Manier (J.-F.), 1950. - Recherches sur les Trichomycètes. Ann. Sci. Nat. (Bot.), 11e série, 11, 53-162 (cf. p. 146).

Schneider (A.), 1876. - Contribution à l'histoire des Grégarines des Invertébrés de Paris et de Roscoff. Arch. Zool. Exp. Gén., 4, 493-604.

- 1882. - Seconde contribution à l'étude des Grégarines. Ibid., 10, 423-450. THÉodoridès (J.), 1955. - Contribution à l'étude des parasites et phorétiques de Coléoptères terrestres. 1 vol., 310 p. Act. Sci. Ind. Hermann, n 1217.

Tuzet (O.) et Gú́rin (R.), 1946. - Recherches sur les Grégarines parasites de quelques Diplopodes du Languedoc. Bull. Biol. Fr. Belg., 80, 466-476.

- et Ormìres (R.), 1956. - Sur quelques Grégarines de la région de Sète. Ann. Parasit. Hum. Comp., 31, 317-330.

Watson (M. E.), 1916. - Studies on Gregarines. Ill. Biol. Monogr., II, 3, 258 p. Weiser (J.), 1954-1955. - Ein Beitrag zur kenntnis der Parasiten des Borkenkäfers Ips typographus L. (en tchèque, résumé allemand), I et II. Acta Soc. Zool. Bohem., 18, 217-224; 19, 374-380.

\section{ADDENDUM}

Ce travail ayant été rédigé en 1957 , plusieurs travaux concernant les parasites qui y sont mentionnés ont paru depuis.

Dans une étude sur les Grégarines de Dytiscides de la région de Clermont-Ferrand, J. Baudoin (1960) signale Legeria agilis (Schneider) chez des larves de Dytiscus marginalis et d'un ? Ilybius sp.,

(6) Pour ne pas alourdir ce tableau, nous n'y donnons pas les noms d'auteurs des espèces (hôtes et parasites), qui figurent dans le texte de cette étude. 
ainsi que chez l'imago de Rhantus suturellus, qui sont des hôtes nouveaux pour cette espèce.

Par contre, Baudoin n'a pas vu le curieux Actinocéphalide que nous décrivons ici.

Pour ce qui est des Nématodes Oxyurides, Thelastoma galliardi Dollfus a été placé dans le genre Cephalobellus par Basir (1956), d'après la position du pore excréteur (post-bulbaire) et la morphologie caudale des deux sexes.

Osche (1959) et Leibersperger (1960) adoptent les vues de Basir et le second de ces auteurs met en outre en synonymie Thelastoma brumpti Théodoridès 1955 avec Ciephalobellus galliardi (Dollfus).

Malgré l'avis de ces auteurs et en accord avec R.-Ph. Dollfus (in litt.), il nous semble que le Thelastomatidx des Diplopodes de Richelieu appartient bien au genre Thelastoma et diffère de $T$. brumpti (parasite d'une larve de Coléoptère Scarabéide), tant morphologiquement que par les hôtes.

(Octobre 1960).

\section{RÉFÉRENCES ADDITIONNELLES}

Basir (M. A.), 1956. - Oxyuroid Parasites of Arthropoda. A monographic study. Zoologica, 38, fasc. 106,79 p., 13 pl. h.t.

Baudorn (J.), 1960. - Contribution à l'étude des Grégarines de Dytiscides. Thèse Doct., 3e cycle, Clermont-Ferrand, 32 p., 14 pl. (ex. ronéotypé). Leibersperger (E.), 1960. - Die Oxyuroidea der europäischen Arthropoden. Parasitol. Schrift., fasc. 11, 150 p.

Osche (G.), 1959. - Systematische, morphologische und parasitophyletische Studien an parasitischen Oxyuroidea (Nematoda) exotischer Diplopoden. Zool. Jahrb. (Syst. Okol.), 87, p. 395-440.

Laboratoire d'Evolution des Etres Organisés de la Faculté des Sciences de Paris et Station Expérimentale de Richelieu (Indre-et-Loire) 